\title{
DESIGN AND CONTROL OF UNDERWATER HYBRID VEHICLE CAPABLE OF PERFORMING NUMEROUS TASKS
}

\section{K. Shahani ${ }^{1}$, C. Wu ${ }^{1}$, R. Persaud ${ }^{2}$ and H. Song ${ }^{1}$}

${ }^{1}$ Ocean Optics Lab, Institute of Ocean Technology, Ocean College, Zhejiang University Hangzhou 310058, China, kamranshahani@zju.edu.cn, chaopengwu@zju.edu.cn, hongsong@zju.edu.cn

${ }^{2}$ Institute of Naval Architecture and Ocean engineering, Ocean College, Zhejiang University Hangzhou 310058, China,persaud rudolph@yahoo.com

\begin{abstract}
:
This paper is about Ocean Strider; an Underwater Hybrid Vehicle that is modified with hybrid (manual and autonomous) control system. The aims concerning this Underwater Hybrid Vehicle are to be competent to operate underwater by using remote control via operator and seek out the user interested objects. Autonomously control system visually follows and manages a secured position comparable to a motionless target and avoids the hindrances for reliable navigation by using vision and ultrasonic sensor. Vision is a fundamental root that promotes the underwater robot to execute various tasks autonomously. Ocean Strider is an intelligent vehicle to explicitly identify, locate and respond perfectly by specifying from distinct color codes and dimension of the objects. Multiple experiments have been conducted in the laboratory to understand manual and autonomous control system which includes grasping, location and traveling pattern with respect to objects.
\end{abstract}

Keywords: Hybrid underwater vehicle, CFD, artificial intelligence, underwater vision, object detection

\section{NOMENCLATURE}

$\begin{array}{llll}L & \text { Length } & V & \text { Velocity Horizontal } \\ W & \text { Width } & B & \text { Battery } \\ H & \text { Height } & P V & \text { Propulsion Vertical } \\ W k g & \text { Weight } & P H & \text { Propulsion Horizontal }\end{array}$

\section{Introduction}

Remotely operated underwater vehicles (ROV) and Autonomous underwater Vehicles (AUV) are currently being utilized for scientific, commercial, domestic and military underwater applications and are revolutionizing the ability to map, monitor, search and rescue in the marine environment (Antonelli et al., 2008). These vehicles required programming and control systems in order to perform underwater tasks to aid in research and development and other purposes as demanded by the owner. Ocean Strider is a hybrid underwater vehicle developed by a team of graduate students at Ocean Opto-Electronics and Automation Lab, Ocean College, Zhejiang University China. The vehicle is with vital payload aptitude, have special-quality video. The mechanical design assures motion levelness for the advanced development of positioning data and payload. Ocean Strider movement is afforded by six self-governing thrusters. With a number of new inventions, Ocean Strider presents a powerful, lighter, and more active platform with advanced capabilities over previous underwater vehicles. Ocean Strider operates in two distinct modes. In autonomous mode, the vehicle can work as AUV competent of inspecting the sea with the image sensor and sonar. For proximate sampling and imaging purpose, ocean strider can be switched at sea to convert into tethered vehicle named as ROV. This design combines a novel technique, tether for large bandwidth, light weight, live video and data telemetry to the exterior, facilitating noble quality remote controlled teleportation by an operator. 
The development of Ocean Strider, a hybrid underwater robot was solely for the purpose of research and competing in the 2017 Oceanology International China underwater robot competition, competition was held on September 2017 in Tianjin, China which attracted participants from different universities across China with an aim to design and develop underwater vehicles to execute missions formed after real-life situations in the marine environment as a result, the team secured 3rd place in the 2017 OI China underwater robotic competition.

As organizations improve underwater operations, including uniform drilling, larger equipment with complicated operations in the seabed that requires modern technology to be reached(Christ and Wernli Sr, 2014). From history researcher face some challenges, oceanographers were performing the experiment with AUV for practice in a research on coral rocks including scientific diving later it was lost to re-surface (2010). However, we evaluate this challenging face could be corrected by designing hybrid underwater vehicle. ROVs make errors most often because their human driver does in consecutive operations such as search and rescue, underwater shipping of accessories and pipeline inspection(Anderson, 1958). To overcome these challenges, we have invented a hybrid underwater vehicle to automate some of the conventional stuff that recently underwater vehicles do, like missing of AUV, our vehicle is hybrid having tether as well so we can retrieve back the vehicle to the surface manually if any problem occurs in autonomous mode. Fig. 1 Show the Ocean Strider with control system.

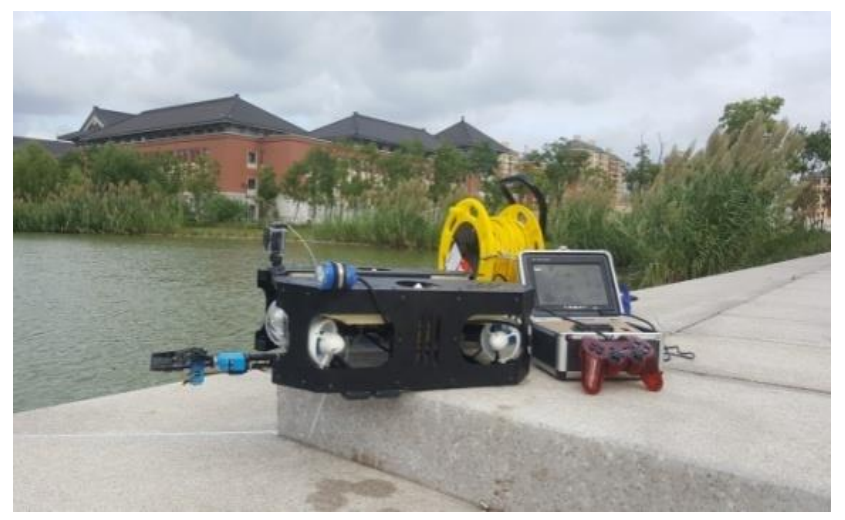

Fig. 1: Ocean Strider and control system.

\section{Methodology}

\subsection{The Ocean Strider vehicle}

Considering the designs of ROV and AUV worldwide and observing vehicles in operation, there were several different basic designs available. For the hybrid underwater vehicle, it is considered in its basic configuration to design a flat- shaped vehicle, after several periods of investigation and study to design a feasible system that can stand quiet and float on site. While the shape of the ocean strider can vary by replacing fascinating modules, the vehicle features are shown in Table 1.

Table 1: Parametric details of numerical models used to study the analysis

\begin{tabular}{|c|c|}
\hline Parameters & Magnitude \\
\hline Length, $L$ & $480 \mathrm{~mm}$ \\
\hline Width, $W$ & $370 \mathrm{~mm}$ \\
\hline Height, $H$ & $160 \mathrm{~mm}$ \\
\hline Weight, $W$ & $7.5 \mathrm{~kg}$ \\
\hline Depth, $D$ & $100 \mathrm{~m}$ \\
\hline Battery, $B$ & Li-Ion $18 \mathrm{Ah}$ \\
\hline Propulsion Vertical, $P V$ & 2 Thrusters \\
\hline Propulsion Horizontal, $P H$ & 4 Thrusters \\
\hline Velocity, $V$ & $0-1.5 \mathrm{~m} / \mathrm{s}$ \\
\hline
\end{tabular}




\subsection{Mechanical structure}

The Ocean Strider's design was based on the Blue ROV. Blue ROV is a small ROV with two waterproof internal solids carrying electronics and batteries, including six thrusters (Aili and Ekelund, 2016). Regarding to the shape of this innovative vehicle, we have created a shape that holds the gripper that allows the objects to be gripped undersea, having noiseless valid hydrodynamics and moderate weight. The structure has the function of holding the thrusters, the waterproof enclosure and all weights. The fundamental design of the frame was to ensure that the water was best discharged through the open frame to reduce drag (Caccia et al., 2000). Choosing the material for the underwater robot frame was a bit complicated. However, a detailed analysis of the selection of materials has been carried out and the most suitable material for the proposed model was a high density polyethylene (HDPE) and aluminum. The advantages associated with the use of these materials are their durability, their dimensional stability (keeping their physical properties underwater) and their structural strength(Tavman, 1996). The ocean strider structure consists of a transparent acrylic hull, aluminum frame, and end caps. To make hull watertight, O- Rings were attached to the end caps. The complete assembly was then developed in Solid Works 2016 CAD software.

Fig. 2 shows the Solid works simulation analysis performed on the Ocean Strider; this study was performed to find out the total Volume of $411.270 \mathrm{in}^{3} \mathrm{~s}$ and total Mass of $7.16 \mathrm{~kg}$.

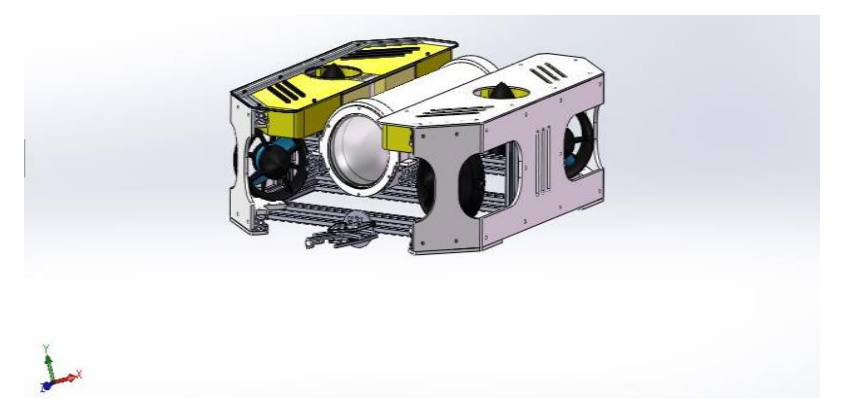

Fig. 2: Ocean Strider Solid works model.

\subsection{Propulsion}

Ocean Strider utilizes propellers and motors to drive inside the water. Such propellers and motor combinations are known as thrusters. Later doing some investigation on the suitable combination of motor and propellers, Blue Robotics thrusters were preferred that will suit the constraints of the Ocean Strider. Ocean Strider has 6 thrusters of Blue Robotics. The thrusters used in this robot are custom made M200 from Blue Robotics, 300W brushless motors with 85 percent average efficiency. Each engine has a nylon 3 blade propeller and has an electronic speed controller of 20 amperes. Due to their compact size and design, brushless motors are more competent and lightweight in the same way as brushed motors.

These thrusters were robust in the Blue ROV and experienced all the tests conducted by the Ocean Strider. These thrusters give the Ocean Strider with a speed of $1.14 \mathrm{~m} / \mathrm{s}$ at full energy.

The investigation from the market for underwater robots shows two different configurations used for horizontal thruster arrangements, the " $\mathrm{H}$ " layout configuration, which only requires three thrusters but has a drawback; it takes bi-directional control for forwarding and reverses driving. The "Vectored" layout configuration uses four unidirectional control thrusters, but its drawback is that it takes the highest thrust for the forward and reverse moment due to the 45 degree offset of each thruster from the direction of a voyage (Omerdic and Roberts, 2004). After a conservative study, the vector design was preferred. The decline in thrust due to the angled thruster configuration was considered an appropriate task for simple electronics and motion directions. For vertical placement with roll axes, two bi-directional thrusters were placed at the top of the left and right sides and are properly installed vertically. 


\subsection{Display screen}

The display screen selected is 7 inch TFT color monitor fixed in top controller box. Screen is powered by the $12 \mathrm{~V}$ onboard controller. Throughout operation of robot, the screen is used to display the following status:

- External temperature $\cdot$ External pressure

- Depth of the robot Direction of robot (compass)

- Heading of the robot $\cdot$ Operation time

- Voltage of the system · Low voltage alert

\subsection{Power management}

Vehicle power is generated by rechargeable Li-Ion batteries stationed in the electronics tray, with an entire energy of $300 \mathrm{Wh}$. depending on vehicle speed and payload conditions, those batteries can serve up to 4 hours. The vehicle is additionally provided with smart power control scheme which is intelligent of managing every feature of battery charging and vehicle powering. In extension, it gives the overall battery energy state broadcast through the tether to top controller screen. To charge the vehicle, a custom 35W DC power supply is used. Batteries can be completely energized in nearly 4 hours.

The robot top controller requires several different voltage levels. The Arduino controller needs an input from $4.5 \mathrm{v}$ to 14.0 Volt, the video screen needs 12 Volt, and PS2 controller needs 5Volts for running. Power is supplied by $16 \mathrm{~V}, 18 \mathrm{Ah}$ battery. 7805 voltage regulator is used to step down the $16 \mathrm{v}$ to $5 \mathrm{v}$ for PS2 and other components'. The robot bottom controller also requires several voltage levels same as top controller we use some regulators and a power distribution board for thrusters.

\subsection{Manual control via PS2}

The top controller use PS2 joystick to allocate the user to direct the pose of the robot individual from the diverse five axes, switch the robot mode auto/manual, on/off the lights, scan the objects, and arm control(Wu et al., 2008). The controller manages the contemporary status of particular joystick or buttons based on the sense stated from the data, generates the states to the appropriate instruction to the robot. Fig. 3 shows the flow diagram for PS2 based manual control.

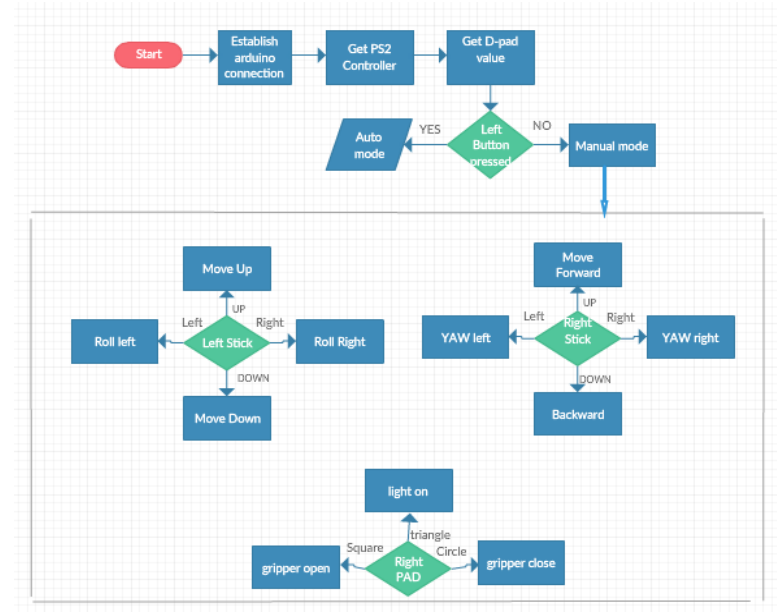

Fig. 3: Flow diagram for manual mode

\subsection{Autonomous control}

To cautiously navigate, ocean strider should be satisfactory to precisely detect hindrances, make immediate and suitable judgments and pick the proper path to bypass them (Pappas and Tzifas). For the navigation system, we are adopting pixy CMU cam5 image sensor in parallelism with the ultrasonic sensor, as image sensor solely doesn't read the data concerning the distance of the target from the vehicle. Consequently, with the use of the 
ultrasonic sensor, we wish to get the data of distance also (Chai et al., 2017, Lee et al., 2012, Al-Khatib et al., 2015, Cruz et al., 2011). Therefore applying both image sensor and ultrasonic we will have the precise information of a target, concerning its dimension, color and the distance of objects from the vehicle (Conte et al., 2002, Chen et al., 2015, Foresti, 2001). The vehicle also has a combined kit of an accelerometer, magnetometer, and gyro for evaluation of vehicle position (pitch, yaw, and roll). Ocean Strider can maintain a secure gap from the target, distinguish such object and take suitable steps if any moving object comes in front of it. It can additionally explore the objects of our interests. Fig. 4 shows the flow diagram for autonomous control.

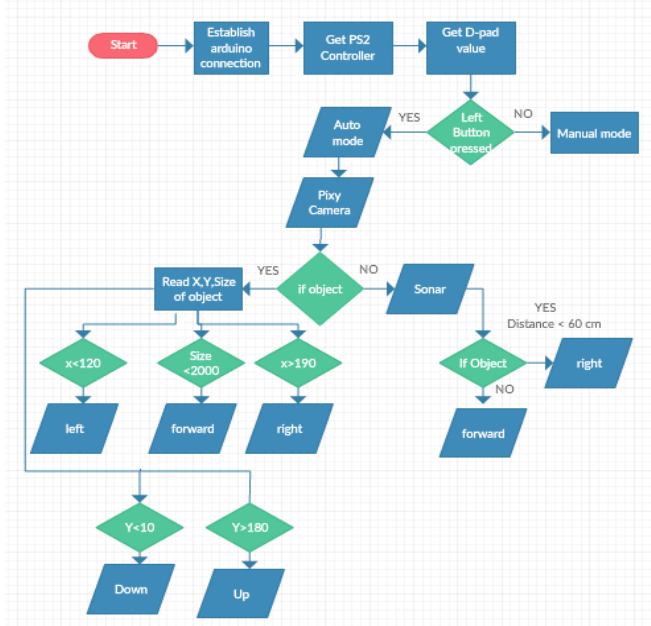

Fig. 4: Flow diagram for autonomous control

\section{Results and Discussion}

The primary water tests with the Ocean Strider have conducted in a laboratory experiment tank in the manual and autonomous modes. The two subsections below conclude the experimental results of the vehicle achieved succes sively.

\subsection{Manual mode}

In manual mode, the vehicle is controlled via a surface controller which displays information of the actual system state, example the depth, temperature, mis sion timing, battery status and has an integrated video screen which displays the video feed off the vehicle. Fig. 5(a) shows the Ocean Strider maneuver in a water tank. The setup consists of the materials located in a tank, the operator manipulates the vehicle through a PS2 controller and inspects the water tank, find and capture the images of the equipment underwater. As with the experiments, ocean strider can also record the high definition videos from the underwater environment which are shown in Fig. 5(b).

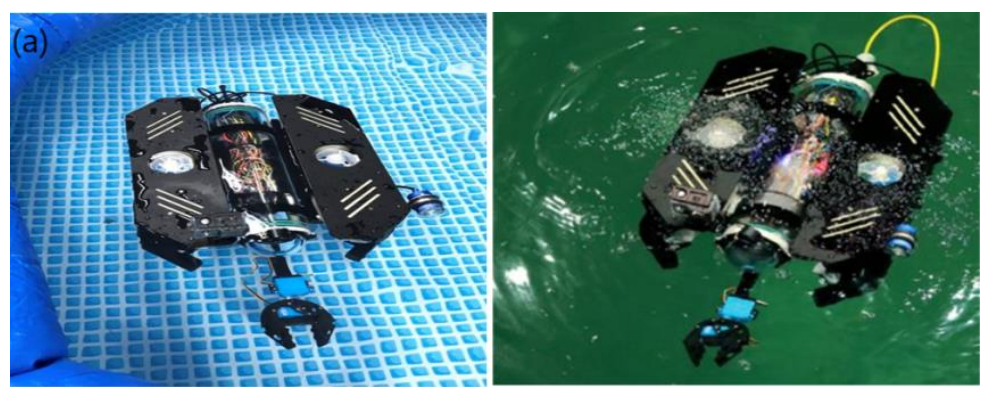

Fig. 5(a):Working in manual mode 


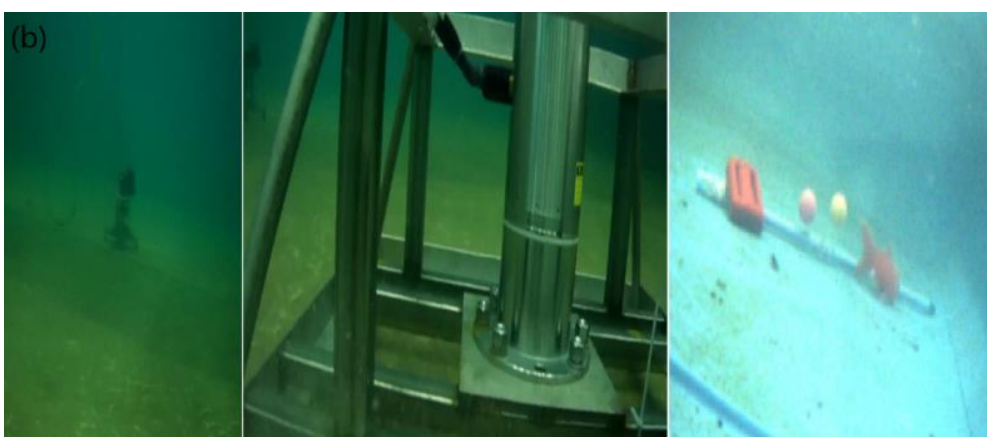

Fig. 5(b): Inspecting in towing tank

\subsection{Autonomous mode}

The autonomous control can be activated or deactivated from the joystick at any time using the particular switch. Later, proving the affirmed method in a manual mode, an attempt was conducted in the water tank in a completely autonomous mode. Many distinct objects were located in the tank, as shown in Fig. 6(a). In the primary examination, the Ocean Strider was only experimented to realize how vast it can track and identify a determined object, and Arduino Plotter was used to achieve the object's X and Y coordinates as shown in Fig. 6(b-c). The image sensor was attached to a computer wirelessly using Bluetooth throughout the experiment with multiple illumination circumstances and PixyMon was practiced to ascertain when the image sensor could recognize the object. The red curve reveals results from a tank while the vehicle does not use its lights in the laboratory with just a few lanterns. The green curve shows the outcome of a vehicle with lights in the tank. Fig. 6 (d) shows the highest distance with changed exposure conditions to the target.

The second test was planned with an ultrasonic sensor to estimate the distance between robot and object. If the image sensor recognizes the object, the ultrasonic sensor will be in hibernation mode and the vehicle will move in the path of the target. Fig. 6(e) displays the output of the image sensor versus the ultrasonic sensor.

The purpose of the Hybrid Underwater Vehicle was to design an underwater vehicle that can be manually controlled with the operator using a surface controller and using vision and ultrasonic sensors a utonomously. The joystick control functionality over tether connection has been manifested. The principal focus of the task here was to expedite the design and performance so that long-range distant control is feasible with lowering drag and noise over the tether. The joystick was used to control the vehicle's maneuvering because it has two sticks and several keys. The results in Fig. 5(b) displays the underwater instruments with a high - quality real-time video throughout underwater exploration in the pool and in the tow tank at a depth of $0.5 \mathrm{~m}$ to $5 \mathrm{~m}$.

The manual mode is not the primary control mode. In order to execute a fully autonomous mode the merging of vision and ultras onic sensors were used. To acquire the exposure value of the image sensor, the highest distance between the Ocean Strider and the object was measured at two different light positions. At the period of analysis, the image sensor was attached to a laptop with PixyMon in order to identify the object. The second experiment was performed to analyze the distance between the Ocean Strider to the object with the help of using an Ultrasonic sensor.

Firstly, the object was kept in front of the Ocean Strider along the center line with no light in the tank. As you can see in Fig. 6(d) there is no light source present in the tank, the image sensor can recognize the object from distance 40 to $55 \mathrm{~cm}$. Therefore it clearly shows the linear line in the graph. The waviness of the graph is due to the moving object while changing the signature of the object at different distances. Secondly, the image sens or has been provided the light source and found that now image sensor can detect the object from distance 30 to 65 $\mathrm{cm}$. later we started decreasing the light source and found that the object detection by image sensor is also decreasing which clearly confirms that any slight changes in light source can also alter the distance and exposures(Znamenskiy and Schmeitz, 2018, Rueggeberg et al., 1994). The test was performed several times by changing the distance between the Ocean Strider and object. However, the vision sensor cannot detect the object at an exposure rate of less than $90 \mathrm{~cm}$. 

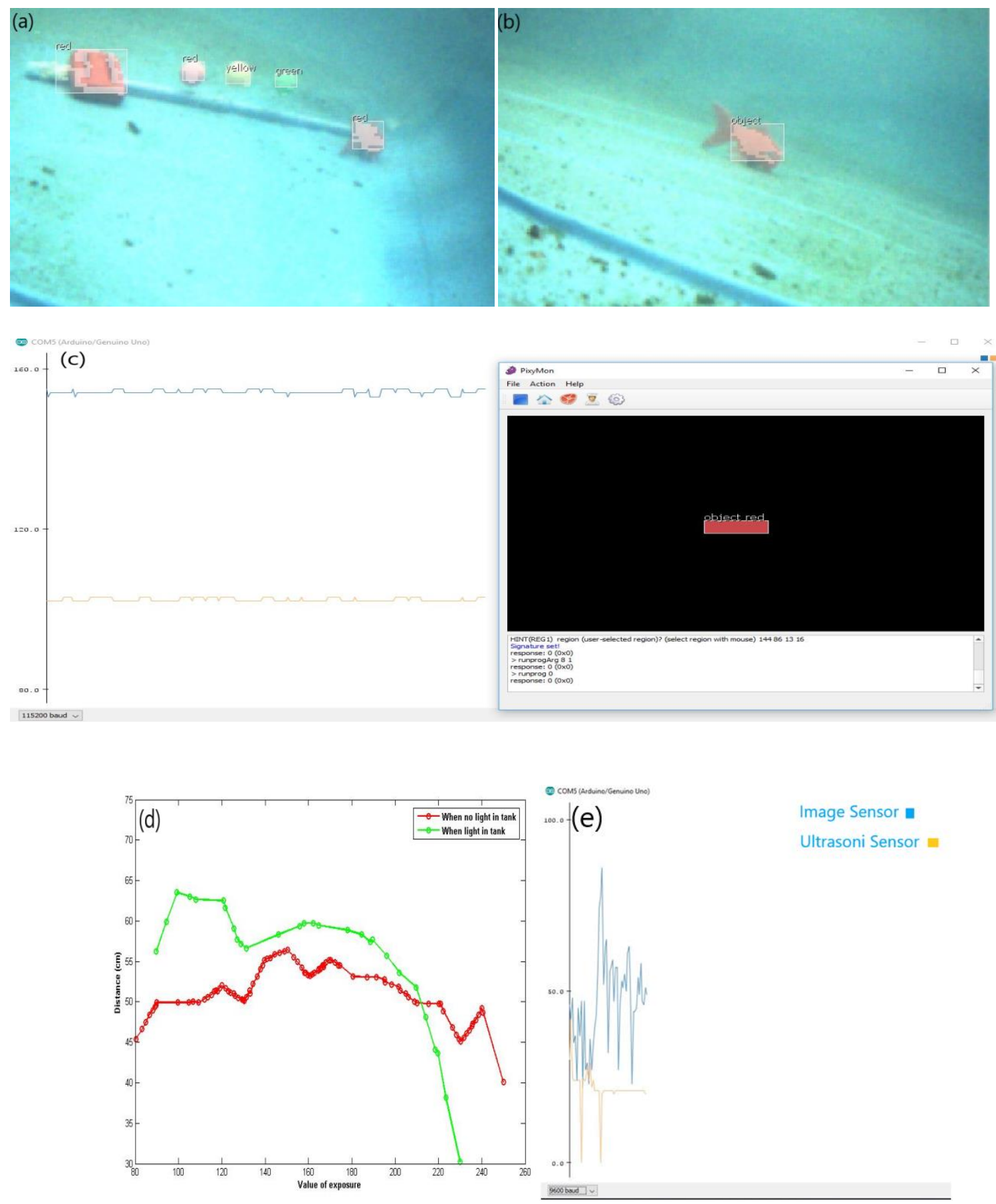

Fig. 6: (a) Multiple objects (b) Defined Object (c) X-Y values of defined object (d) Distance at different exposures (e) Image sensor VS Ultrasonic sensor output

\section{Conclusion}

This research demonstrates the wide control and design of a unique hybrid underwater vehicle, which includes two control methods viz. manually with joystick and autonomous control with the fusion of ultrasonic and vision image sensorsystems. The results achieved have strengthened the effectiveness of the mechatronic design and control techniques. Numerous experiments confirmed that the Hybrid Underwater Vehicle has been able to perform numerous tasks manually and autonomously. 
The results show that the detection and precession of objects in the vehicle could be improved by using fast image sensors. The ultrasonic sensor can process within $5 \mathrm{~m}$, while vision sensors are lacking in real time detection as distance increases.

\section{Acknowledgements}

The authors gratefully acknowledge the research facilities and financial support for the Ocean Strider provided by Institute of Ocean Technology, Ocean College, Zhejiang University China.

\section{References}

Aili, A. and Ekelund, E. (2016): Model-based design, development and control of an underwater vehicle, M.Sc. Thesis, Automatic Control Department of Electrical Engineering, Linköping University, Sweden. https://liu.diva-portal.org/smash/get/diva2:939494/FULLTEXT01.pdf

Al-Khatib, H., Antonelli, G., Caffaz, A., Caiti, A., Casalino, G., De Jong, I. B, Kebkal, K. (2015): Navigation, guidance and control of underwater vehicles within the widely scalable mobile underwater sonar technology project: an overview, IFAC-Papers OnLine, Vol. 48, No. 2, pp. 189-193.

https://doi.org/10.1016/j.ifacol.2015.06.031

Anderson, P. D. (1958): Psychological aspects of machine and work place design, M.Sc. Thesis, Dept. of Industrial Engineering and Industrial Arts, Kansas State College of Agriculture and Applied Science, USA. http://krex.k-state.edu/dspace/bitstream/handle/2097/23160/LD2668R41958A53.pdf

Antonelli, G., Fossen, T. I. and Yoerger, D. R. (2008): Underwater Robotics, Springer Handbook of Robotics, pp. 987-1008. https://doi.org/10.1007/978-3-540-30301-5 44

Caccia, M., Indiveri, G. and Veruggio, G. (2000): Modeling and identification of open-frame variable configuration unmanned underwater vehicles, IEEE Journal of Oceanic Engineering, Vol. 25, No. 2, pp. 227240. https://doi.org/10.1109/48.838986

Chai, X., Gao, F., Qi, C., Pan, Y., Xu, Y. and Zhao, Y. (2017): Obstacle avoidance for a hexapod robot in unknown environment, Science China Technological Sciences, Vol. 60, No. 6, pp. 818-831. https://doi.org/10.1007/s 11431-016-9017-6

Chen, H.H., Chuang, W.N. and Wang, C.C. (2015): Vision-based line detection for underwater inspection of breakwater construction using an ROV, Ocean Engineering, Vol. 109, pp. 20-33. https://doi.org/10.1016/j.oceaneng.2015.09.007

Christ, R. D. and Wernli Sr, R. L. (2014): The ROV manual: a user guide for remotely operated vehicles, Butterworth-Heinemann.

Conte, G., Zanoli, S. and Scaradozzi, D. (2002): An automatic guidance system for a small work-class ROV, IFAC Proceedings Vol. 35, No. 1, pp. 67-72. https://doi.org/10.3182/20020721-6-ES-1901.00823

Cruz, N. A., Matos, A. C., Almeida, R. M., Ferreira, B. M. and Abreu, N. (2011): Trimares-a hybrid AUV/ROV for dam inspection, Presented at the OCEANS 2011. https://doi.org/10.23919/OCEANS.2011.6107314

Foresti, G. L. (2001): Visual inspection of sea bottom structures by an autonomous underwater vehicle, IEEE Transactions on Systems, Man, and Cybernetics, Part B (Cybernetics), Vol. 31, No. 5, pp. 691-705. https://doi.org/10.1109/3477.956031

Lee, D., Kim, G., Kim, D., Myung, H. and Choi, H.-T. (2012): Vision-based object detection and tracking for autonomous navigation of underwater robots, Ocean Engineering, Vol. 48, pp. 59-68. https://doi.org/10.1016/i.oceaneng.2012.04.006

Omerdic, E. and Roberts, G. (2004): Thruster fault diagnosis and accommodation for open-frame underwater vehicles, Control engineering practice, Vol. 12, No. 12, pp. 1575-1598. https://doi.org/10.1016/j.conengprac.2003.12.014

Pappas, S. O. and Tzifas, G. P. (2013): Wireless control vehicle via Playstation 2 controller, World Transactions on Engineering and Technology Education, Vol. 11, No. 3, pp. 348-352.

Tavman, I. (1996): Thermal and mechanical properties of aluminum powder-filled high-density polyethylene composites, Journal of Applied Polymer Science, Vol. 62, No. 12, pp. 2161-2167. https://doi.org/10.1002/(SICI) 1097-4628(19961219)62:12<2161::AID-APP19>3.0.CO;2-8

Wu, J., Chen, S. and Liu, D. (2008): Control and power electronics technology in renewable energy, Science in China Series E: Technological Sciences, Vol. 51, No. 6, pp. 702-712. http://dx.doi.org/10.1007/s 11431-0080064-2

Whoi (2010): Pioneering deep-sea robot lost at sea, Woods Hole Oceanographic Institution, News Release, Accessed on 3 June 2018. http://www.whoi.edu/page.do?pid=7545\&tid=7342\&cid=70487. 